\title{
5 Zusammenfassung
}

Die Investitionstheorie dient der Unterstützung realer betriebswirtschaftlicher Entscheidungen unter realistischen Bedingungen. Die Grenze der Zahlungsbereitschaft - bspw. beim Kauf einer Eigentumswohnung - nennt man Entscheidungswert. Er hängt von den finanziellen Vorteilen ab, die man zukünftig aus dem Bewertungsobjekt erwartet. ${ }^{749}$ Weil echtes Geld auf dem Spiel steht, sind hypothetisch-idealisierte Laborbedingungen unbrauchbar. ${ }^{750}$ Ein für Immobilien geeignetes Bewertungsmodell muß trotz abstrahierender Vereinfachungen den unvollkommenen Kapitalmarkt als realitätsnahe Modellumgebung berücksichtigen. Während die Hauptaufgabe der theoretischen Bewertung darin liegt, Zahlungsströme in einen Wert umzuformen, ${ }^{751}$ hängt die Qualität des ermittelten Wertes wesentlich von den bei der Berechnung verwendeten Eingangsdaten ab. ${ }^{752}$ Deren Zusammenstellung erfordert eine differenzierte Auseinandersetzung mit dem Bewertungsgegenstand.

Bewertungsobjekte vom Typ Eigentumswohnung bringen zahlreiche Besonderheiten mit sich, die der quantitativen Bewertungstheorie nur bedingt zugänglich sind, aber große Auswirkungen auf den finanziellen Zukunftserfolg haben können. Sie sind u.a. auf den ausgeprägten Unikatscharakter von Immobilien, auf das soziale Miteinander der Wohnungseigentümer und auf die speziellen Regelungen des Wohnungseigentumsrechts zurückzuführen.

Die Beurkundung des Kaufvertrags kann gewissermaßen als Türschwelle zum Wohnungseigentum gesehen werden, doch selten wird der Notar umfangreiche Aufklärungsarbeit über diese ,neue Eigentumsform“"753 leisten. Lückenhafte Kenntnisse über die Besonderheiten des Wohnungseigentumsrechts bergen Konfliktpotential. Woher soll ein gewöhnlicher Wohnungseigentümer z.B. wissen, daß er Sachbeschädigung begeht, wenn er sich ohne Einbezug der Miteigentümer zur Erneuerung der Fenster seiner Wohneinheit entscheidet? ${ }^{754}$ Schlimmstenfalls führen Mißverständnisse zu langwierigen Grabenkämpfen innerhalb einer Eigentümergemeinschaft und verursachen nicht selten wertmindernde Anwalts- und Gerichtskosten. Rund 22.500 gerichtlich ausgetragene WEG-Binnenstreitigkeiten allein im Jahr 2017 zeugen vom Konfliktpotential, welches der Rechtsform innewohnt. ${ }^{755}$

\footnotetext{
${ }^{749}$ Vgl. BUSSE VON COLBE (1957), S. 42.

${ }^{750} \mathrm{Vgl}$. HAX (1964), S.430, TOLL/HERING (2017), S. $461 \mathrm{f}$.

${ }^{751} \mathrm{Vgl}$. HERING (2014), S. 4 f.

${ }^{752} \mathrm{Vgl}$. BRÖSEL (2002), S. 74.

${ }^{753}$ SAUREN (2014a), Rn. 2.

${ }^{754}$ Vgl. § 303 Abs. 2 StGB.

${ }^{755}$ Vgl. STATISTISCHES BUNDESAMT (2018c), S. 38.

(C) Der/die Autor(en) 2021
}

S. Walochnik, Bewertung von Eigentumswohnungen, Finanzwirtschaft, Unternehmensbewertung \& Revisionswesen, https://doi.org/10.1007/978-3-658-30840-7_5 
Manchmal überfordern größere Instandhaltungsmaßnahmen Verwalter und Eigentümergemeinschaft - nicht nur finanziell. Anstatt einen nach Stundenhonorar bezahlten Fachplaner mit einer gründlichen Diagnose und später auch Planung der Maßnahme zu beauftragen, verwechseln manche kostensensitiven Kapitalanleger den Verwalter plötzlich mit einem Hochbauingenieur. ${ }^{756}$ Sie erwarten von ihm die kosten- und ziellose Beschaffung von drei, vier oder besser fünf Reparaturangeboten. ${ }^{757}$ Bei überschaubaren Vorgängen wie dem Anstrich eines Treppenhauses mag das sinnvoll sein, aber bei komplexeren Maßnahmen sind die Ausführungsvorschläge der Handwerker selten vergleichbar. ${ }^{758}$ Wohnungseigentümergemeinschaften stolpern damit von einem Angebot zum nächsten, während die Instandhaltungsrücklage leer bleibt und die Substanz vor sich hin bröckelt - und mit ihr die Vermietbarkeit der Eigentumswohnung.

Die Investitionstheorie bietet keine multifunktionale Patentlösung, jedoch mit der Nutzwertanalyse ein Werkzeug, das bereits bei der Vorselektion potentieller Investitionsobjekte gute Dienste leisten kann. Hier trennt sich die Spreu vom Weizen: Der Zukunftserfolg der Eigentumswohnung steht und fällt mit der Fähigkeit von Verwalter und Eigentümern, Probleme anzupacken und zu lösen, anstatt sich über Jahre hinweg gegenseitig zu blockieren. Für den Investor gehört es zwingend zur Analyse des Bewertungsobjekts, auf charakterliche Merkmale von Verwalter und Eigentümern zu achten, weil er in vielen Fällen auf deren Mitwirkung angewiesen ist. ${ }^{759}$ Diese kann er aber nirgends ablesen, sondern benötigt eine Menge Erfahrung und Menschenkenntnis. Dennoch darf bezweifelt werden, daß eine treffende Einschätzung der anderen Akteure selbst bei eingehender Analyse möglich ist. Der präsumtive Käufer kann aber zumindest nach Warnzeichen suchen, die er ggf. in der Beschlußsammlung oder in Protokollen vergangener Eigentümerversammlungen findet. ${ }^{760}$ Wurden Reparaturen aus Geldnot oder Unverständnis abgelehnt oder vertagt, sind größere Probleme absehbar. Anhaltspunkte, ob der Verwalter sein Handwerk versteht, kann die Jahresabrechnung liefern, weil sie die Einhaltung vieler sehr spezieller Formalien fordert. ${ }^{761}$ Um Rückschlüsse aus Vergangenheitsdaten für die Zukunft zu ziehen, sind Intuition, Erfahrung und Fingerspitzengefühl

${ }^{756}$ Vgl. VON HAUFF (2017b), S. 38 f., S. 46.

${ }^{757}$ Vgl. ENGELHARDT (2017a), Rn. 44, CASSER (2018), DRASDO (2018a).

${ }^{758}$ Vgl. MATHAR (2017), S. 260 f.

${ }^{759}$ Flexibilität und Risikobegrenzung besitzt in diesem Kontext hohen Stellenwert. Um die Wohneinheit schlimmstenfalls kurzfristig wieder verkaufen zu können, kann es empfehlenswert sein, bei der Kreditaufnahme zunächst eine variable Verzinsung zu wählen, damit die Bank bei Verkauf keine Vorfälligkeitsentschädigung verlangen kann, vgl. HELLERFORTH (2008), S. 87, RENNERT (2012), S. 122 f., SCHULZE/STEIN/TIETGEN/MÖLLER (2017), S. 65.

${ }^{760} \mathrm{Vgl}$. PFLÜGL (2017e), Rn. 20 ff.

${ }^{761}$ Vgl. dazu CASSER/SCHULTHEIS (2017a), CASSER/SCHULTHEIS (2017b), WANDERER (2017b). Eine Umfrage des Berufsverbands DDIV zeigt, daß man selbst bei einem hauptberuflichen Verwalter keineswegs selbstverständlich von dessen Professionalität oder Kenntnis derartiger Vorschriften ausgehen kann, vgl. DACHVERBAND DEUTSCHER IMMOBILIENVERWALTER E.V. (2017). 
wesentliche Faktoren - und dennoch kann sich im nachhinein herausstellen, daß man falsch lag. ${ }^{762}$

Auch die Vermietbarkeit wird von einer Vielzahl von Merkmalen beeinflußt, die zwar beobachtbar, aber kaum meßbar sind. Man denke nur an die zahlreichen Faktoren, die bei Neuvermietung auf die Zahlungsbereitschaft von Mietinteressenten einwirken, wie z.B. eine gute Verkehrsanbindung, fußläufig erreichbare Geschäfte oder den günstig geschnittenen Grundriß. Der mit der Standortgebundenheit einhergehende Unikatscharakter schränkt die Vergleichbarkeit ein ${ }^{763}$ - und damit auch die Anwendbarkeit von Durchschnittswerten für Miete, Instandhaltungs- und Betriebskosten. Meist handelt es sich um statistische Vergangenheitsdaten, die aus einem großen Fundus an Wohnungen gewonnen wurden und kaum zu den individuellen Gegebenheiten eines bestimmten Bewertungsobjekts passen dürften.

Nachdem der Investor den Zukunftserfolg einer Eigentumswohnung prognostiziert hat, muß ihm klar sein, daß die erwarteten Mietüberschüsse noch durch die Einkommensteuerpflicht geschmälert werden. Wenn er, wie 80 Prozent der deutschen Wohnimmobilieneigentümer, eine natürliche Person ist, ${ }^{764}$ ergibt sich sein Steuersatz erst aus der Gesamtbetrachtung aller Einkommensquellen - dies erfordert den Einsatz von Totalmodellen. ${ }^{765}$ Solche sind jedoch meist linear und damit inkompatibel zur Steuerlast, deren Höhe nach deutschem Recht mit einer nichtlinearen Steuertariffunktion berechnet wird.

Ziel der vorliegenden Arbeit war die Bewertung einer Eigentumswohnung mit Hilfe des investitionstheoretischen Zustands-Grenzpreismodells. Um den Gegebenheiten der Bewertungssituation bestmöglich gerecht zu werden, war dessen Weiterentwicklung erforderlich, um es in die Lage zu versetzen, die nichtlineare Einkommensteuertariffunktion zu berücksichtigen. Ferner erforderten die Besonderheiten dieser Immobilienform, die Auswirkungen vieler, teils schwer quantifizierbarer Merkmale auf den Zahlungsstrom zu erfassen.

In Kapitel zwei wurde die Bewertung sowohl in ihrer allgemeinen Vorgehensweise als auch anhand einer beispielhaften Eigentumswohnung demonstriert. Zur Gewinnung von Eingangsdaten wurden Durchschnittswerte für Mieteinnahmen, Betriebs- und Instandhaltungskosten zusammengestellt und per Nutzwertanalyse an das Bewertungsobjekt angepaßt. Durch Gegenüberstellung mit einer fiktiven, durchschnittlichen Vergleichswohnung wurden aus den Merkmalen des Bewertungsobjekts zuerst Nutzwerte und aus

\footnotetext{
${ }^{762} \mathrm{Vgl}$. HERING (2017), S. 12.

${ }^{763} \mathrm{Vgl}$. OLBRICH (2003), S. 348.

${ }^{764}$ Vgl. dazu Unterabschnitt 3.2.2.1.

${ }^{765}$ Vgl. ADAM (2000), S. 163, JAPES (2011), S. 13 f., HENSELMANN/KNIEST (2015), S. 319.
} 
diesen schließlich Faktoren zur Individualisierung von Durchschnittswerten abgeleitet. ${ }^{766}$ Die einfließenden Merkmale wurden kategorisiert und mehrere Nutzwerte ermittelt, weil jeder vermietende Wohnungseigentümer durch WEG und Mietvertrag zweiseitig gebunden ist.

Das investitionstheoretische ZGPM erwies sich als geeignetes Bewertungsmodell ${ }^{767}$ und war in der Lage, neben dem Bewertungsobjekt auch das Entscheidungsfeld mit allen immobilienspezifischen Besonderheiten vor dem Hintergrund des unvollkommenen Kapitalmarkts zu erfassen. Die Bewertung erfolgte zunächst - wie in der Literatur bislang üblich - ohne Berücksichtigung von Einkommensteuern. Die mit der theoretischen Exaktheit einhergehende Komplexitätsexplosion, die insbesondere in Großunternehmen auftritt, erwies sich in dem auf Privatpersonen zugeschnittenen Modell als beherrschbar. ${ }^{768}$ Die Objekte und Nebenbedingungen wurden in Form von linearen Optimierungsansätzen aufgestellt, für deren Lösung neben dem in Microsoft Excel integrierten Solver u.a. mit Matlab oder dem hier verwendeten Lingo eine ganze Reihe kostenfreier Programme zur Verfügung steht, die an jedem Arbeitsplatzrechner einsetzbar sind. ${ }^{769}$

Aus den Ergebnissen des ZGPM konnte man nicht nur den Entscheidungswert ablesen, sondern auch Informationen darüber, welche Investitions- und Finanzierungsobjekte in welcher Höhe im optimalen Programm vorkommen. Der Veranschaulichung diente insbesondere ein tabellenförmiger VOFI, der einer differenzierten Vermögensübersicht ähnlich ist. Neben den periodenspezifischen Geldbewegungen konnte auch der Kontostand am Ende jeder Periode einfach abgelesen werden.

In Kapitel drei wurde das ZGPM weiterentwickelt, damit es zusätzlich in der Lage ist, persönliche Einkommensteuern zu berücksichtigen. Nun wurde zu jedem investitionstheoretischen Zahlungsstrom ein steuerliches Schattenobjekt modelliert, um dessen abweichende steuerliche Bemessungsgrundlage abzubilden. ${ }^{70}$ Die grundsätzliche Inkompatibilität zwischen linearem Modell und nichtlinearer Steuertariffunktion wurde überwunden, indem die nichtlineare Steuertariffunktion durch abschnittsweise Linearisierung modelltauglich gemacht wurde. Um den Rechenaufwand des mehrperiodigen Modells einerseits so gering wie möglich zu halten und gleichzeitig die Approximationsgüte bestmöglich zu wahren, wurden zuvor Untersuchungen zur geeigneten Anzahl und Lage der Interpolationsgrenzen angestellt.

Beim Vergleich der Modellergebnisse mit und ohne Steuern zeigte sich bei sonst gleichen Rahmenbedingungen die drastische Auswirkung von Einkommensteuern auf den Entscheidungswert. Die periodische Entnahmemöglichkeit sank um knapp 400 EUR -

${ }^{766} \mathrm{Vgl}$. TOLL/WALOCHNIK (2013), S. 22 f.

${ }^{767} \mathrm{Zu}$ diesem vgl. HERING (2000a).

${ }^{768}$ Vgl. dazu TOLL/WALOCHNIK (2013), S. 35, HERING (2017), S. 149 f.

${ }^{769} \mathrm{Zu}$ rechnergestützten Programmen zur Modellierung von Optimierungsansätzen vgl. WERNERS (2013), S. 123 f., SUHL/MELLOULI (2013), S. 77 ff., DOMSCHKE/DREXL/KLEIN/SCHOLL (2015), S. $14 \mathrm{ff}$.

${ }^{770}$ Vgl. HERING (1998), ergänzend HERING/KLINGELHÖFER (1998). 
und der Entscheidungswert um rund 37.000 EUR. Bei der Interpretation dieser Beträge ist zu beachten, daß die vorangegangene Situation ohne Steuern unvollständig war, weil es sie in der realen Welt nicht gibt. Wenn man bedenkt, daß die Investitionstheorie der Unterstützung realer Entscheidungen dient, hätte der Investor ohne Berücksichtigung von Steuern schlimmstenfalls einen Kaufpreis von 127.728 EUR akzeptiert, verglichen mit seinem eigentlichen Grenzpreis von 90.431 EUR. Damit wäre fälschlicherweise die Hoffnung auf einen zusätzlichen Vermögenszuwachs von rund 37.000 EUR geweckt worden, der aufgrund der Steuerpflicht der Finanzverwaltung zugeflossen wäre. Nur durch Berücksichtigung der Einkommensteuerpflicht erlangt das Modellergebnis eine hinreichende Aussagekraft im Rahmen der Entscheidungsfunktion.

In Kapitel vier wurde das Modell durch insgesamt acht Variationen an verschiedene Spezialfälle angepaßt, die bei Immobilieninvestitionen regelmäßig auftauchen. In unterschiedlichen Szenarien wurden Berechnungen durchgeführt, die wegen veränderter Rahmenbedingungen erwartungsgemäß zu völlig anderen Entscheidungswerten führten. Es ist nicht die Aufgabe von Entscheidungsmodellen, Äpfel und Birnen vergleichbar zu machen; für Entscheidungen in unterschiedlichen Situationen braucht man unterschiedliche Werkzeuge. Die Varianten dienten in erster Linie dazu, mit seiner flexiblen Anpassungsfähigkeit eine der Stärken des ZGPM vorzuführen. ${ }^{771}$ Schließlich läßt sich auch die Realität nicht in eine vorgestanzte Form pressen. ${ }^{772}$ Durch meist nur geringfügige Umformulierung einzelner Nebenbedingungen war das steuerliche Grundmodell auch in ganz anderen Bewertungssituationen anwendbar.

Die in den verschiedenen Szenarien verfolgten Ziele waren unterschiedlich: Beispielsweise dienten die größeren Erhaltungsaufwendungen in Unterkapitel 4.6 der Substanzerhaltung der Mietsache, während die Modernisierungsmaßnahmen in Unterkapitel 4.7 eine Substanzverbesserung mit sich brachten und die Vereinbarung einer höheren Miete zuließen - obwohl private Kleinvermieter selten von Mieterhöhungen Gebrauch machen und sich häufig ein gutes Verhältnis zum Mieter wünschen. ${ }^{773}$ Vom Gemeinschaftseigentum ausgelöste Kollateralschäden am Sondereigentum sind zumindest nicht alltäglich. In Unterkapitel 4.5 dienten sie vor allem dem Zweck, große steuerliche Verluste herzuleiten. Die steuerliche Situation zusammenveranlagter Eheleute und Kapitalgesellschaften in den letzten beiden Unterkapiteln waren schon deswegen nicht mit dem Ausgangsfall vergleichbar, weil allein durch unterschiedliche steuerliche Regelungen völlig andere Ergebnisse entstanden - selbst bei identischem Zahlungsstrom und sonst gleichen Rahmenbedingungen.

Aus dem Vergleich der Modellergebnisse müssen aber auch keine Rückschlüsse gezogen werden. Es ist Aufgabe der Investitionstheorie, Modelle zu liefern, die dem Einzelfall angepaßt werden können, und deren Ergebnisse situativ zu würdigen. Oft ergeben

\footnotetext{
${ }^{771}$ Vgl. BRÖSEL/MATSCHKE (2004), S. 59 f., INWINKL/SCHNEIDER (2008), S. 277, KEUPER/PAPE (2011), S. 328 .

772 Vgl. HAX (1964), S. 430, HERING/VINCENTI (2004), S. 343.

${ }^{773} \mathrm{Vgl}$. SCHULZE/STEIN/TIETGEN/MÖLLER (2017), S. 124.
} 
sich unerwartete Änderungen und erfordern Entscheidungen. Selbst wenn plötzlich der sprichwörtliche Wasserhahn tropft, verändert sich bereits das Entscheidungsfeld. Modelle müssen daher flexibel sein. Einem Investor, der über den Erwerb einer Eigentumswohnung nachdenkt, wurden viele Werkzeuge an die Hand gegeben, um sich ein eigenes, zum Einzelfall passendes Modell zusammenzustellen.

Open Access Dieses Kapitel wird unter der Creative Commons Namensnennung 4.0 International Lizenz (http://creativecommons.org/licenses/by/4.0/deed.de) veröffentlicht, welche die Nutzung, Vervielfältigung, Bearbeitung, Verbreitung und Wiedergabe in jeglichem Medium und Format erlaubt, sofern Sie den/die ursprünglichen Autor(en) und die Quelle ordnungsgemäß nennen, einen Link zur Creative Commons Lizenz beifügen und angeben, ob Änderungen vorgenommen wurden.

Die in diesem Kapitel enthaltenen Bilder und sonstiges Drittmaterial unterliegen ebenfalls der genannten Creative Commons Lizenz, sofern sich aus der Abbildungslegende nichts anderes ergibt. Sofern das betreffende Material nicht unter der genannten Creative Commons Lizenz steht und die betreffende Handlung nicht nach gesetzlichen Vorschriften erlaubt ist, ist für die oben aufgeführten Weiterverwendungen des Materials die Einwilligung des jeweiligen Rechteinhabers einzuholen.

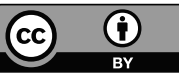

